\title{
Mechanisms of melanoma resistance to treatment with BRAF and MEK inhibitors
}

\author{
Ewa Bartnik², Michał Fiedorowicz², Anna M. Czarnecka²,3
}

IInstitute of Genetics and Biotechnology, Faculty of Biology, University of Warsaw, Poland

2Department of Experimental Pharmacology, Mossakowski Medical Research Centre, Polish Academy of Sciences, Warsaw, Poland ${ }^{3}$ Department of Soft Tissue/Bone Sarcoma and Melanoma, Maria Sklodowska-Curie Institute — Oncology Center, Warsaw, Poland

Several mechanisms of resistance to inhibition of BRAF activity in melanoma cells have been described so far. Genetic studies have shown that mutations in MEK1 kinase (MAP kinase kinase), which result in constitutive activation of ERK kinase, result in resistance to treatment. Another mechanism of the acquired BRAF inhibition resistance is the accumulation of activating mutations in the NRAS oncogene, which drives the activation of CRAF. This in turn leads to a permanent activation of the signal transduction to MEK and ERK. Another important mechanism of resistance is the formation of variants of the BRAFV600E gene splicing, including variants that lack exons 4 to 8 containing the RAS-binding domain. The presence of the p61 BRAFV600E variant leads to the constitutive ERK signal, which is resistant to RAF inhibition. In addition, treatment resistance is affected by hyperactivation of tyrosine kinase receptors such as platelet-derived factor receptor $\beta$ (PDFR $\beta$ ), insulin-like growth factor 1 receptor (IGF-1R) and erythropoietin-producing hepatocellular receptors (EPH) - leading to the induction of the 3-phosphoinositol kinase pathway (PI3K) in patients treated with BRAF or MEK inhibitors. Another interesting path of BRAFi/MEKi resistance is over-expression of the epidermal growth factor receptor (EGFR) through negative feedback in patients treated with BRAF inhibitors (BRAFi) - EGFR is not normally expressed in untreated melanomas.

NOWOTWORY J Oncol 2019; 69, 3-4: 133-141

Key words: BRAF, MEK, melanoma, MAPK, drug resistance

\section{Introduction}

Before the discovery of activating mutations in the BRAF gene, melanoma was considered to be the skin cancer with the worst prognosis. Classical dacarbazine-based chemotherapy regimens provided few therapeutic options. In 2002, in a breakthrough study conducted by the Cancer Genome Project at the Sanger Institute, BRAF [1] mutations were identified in over $60 \%$ of melanoma patients. Currently, melanoma is responsible for over $80 \%$ of skin neoplasm deaths, although its cases account for only $1 \%$ of all skin neoplasm cases. Until 2011, when the Food and Drug Administration (FDA) approved vemurafenib (the first drug acting selectively on a specific mutant protein in the signalling pathway of BRAF/MEK), there was virtually no effective therapy for patients with melanoma with metastases. The group of drugs that include vemurafenib (as well as dabrafenib and encorafenib) is referred to as BRAF (BRAFi) protein inhibitors [2].

The RAS/MAPK pathway (fig. 1) is one of the best known pathways of signal transduction from the cell environment to the cell nucleus in which, as a result of the action of these signals, the transcription of genes associated with the processes of cell growth, division and differentiation is initiated. It can be said that it is a cascade of phosphorylation of subsequent proteins (Ras-Raf-MEK-ERK), where an activated protein phosphorylates a subsequent protein and thus the signal is transmitted up to the transcription factors (among others 
c-Myc and (REB). Activated Ras stimulates RAF kinase protein kinase activity, RAF kinase phosphorylates and activates MEK (MEK1 and MEK2), and MEK phosphorylates and activates mitogen-activated protein kinase (MAPK). RAF and ERK (also known as MAPK) are serine/threonine protein kinases and MEK is a serine/tyrosine/threonine kinase. This signalling pathway is activated by growth factors, hormones and cytokines that interact with the membrane receptor with tyrosine kinase activity (RTK). As a result of these interactions, the membrane receptor is phosphorylated and binds several different proteins, which in turn transmit a signal to the RAS (rat sarcoma) protein. RAS protein then binds guanosine triphosphate, which activates it and in turn transmits the signal to the whole next cascade of protein kinases activated by mitogens (mitogen activated protein kinases - MAPK). The first protein in this pathway is RAF (rapidly accelerated fibrosarcoma protein): BRAF or CRAF. RAF proteins have serine/threonine kinase activity and phosphory- late and activate MAPK kinase, also known as MEK - MAPK/ERK, which in turn phosphorylates another ERK kinase (extracellular signal regulated kinase), which after phosphorylation moves to the cell nucleus, where it activates target transcription factors through phosphorylation. Disturbances in this pathway can lead to neoplasms on the one hand, and several human malformations on the other [3].

Disturbances in this pathway, including oncogenic ones, may occur at different levels as a result of gene mutations - the RAS gene is one of the most frequently mutated oncogenes in human cancers [4]. Disturbances in the whole RAS/ /MAPK pathway occur in $98 \%$ of melanomas [5]. About $50 \%$ of these cancers (but for comparison, only $7-10 \%$ of all cancers in general) have a mutation in the BRAF gene. On the other hand, $80-90 \%$ of these mutations are of the V600E missense type, where amino acid number 600 is glutamic acid and not valine found in wild-type protein. This mutation changes the

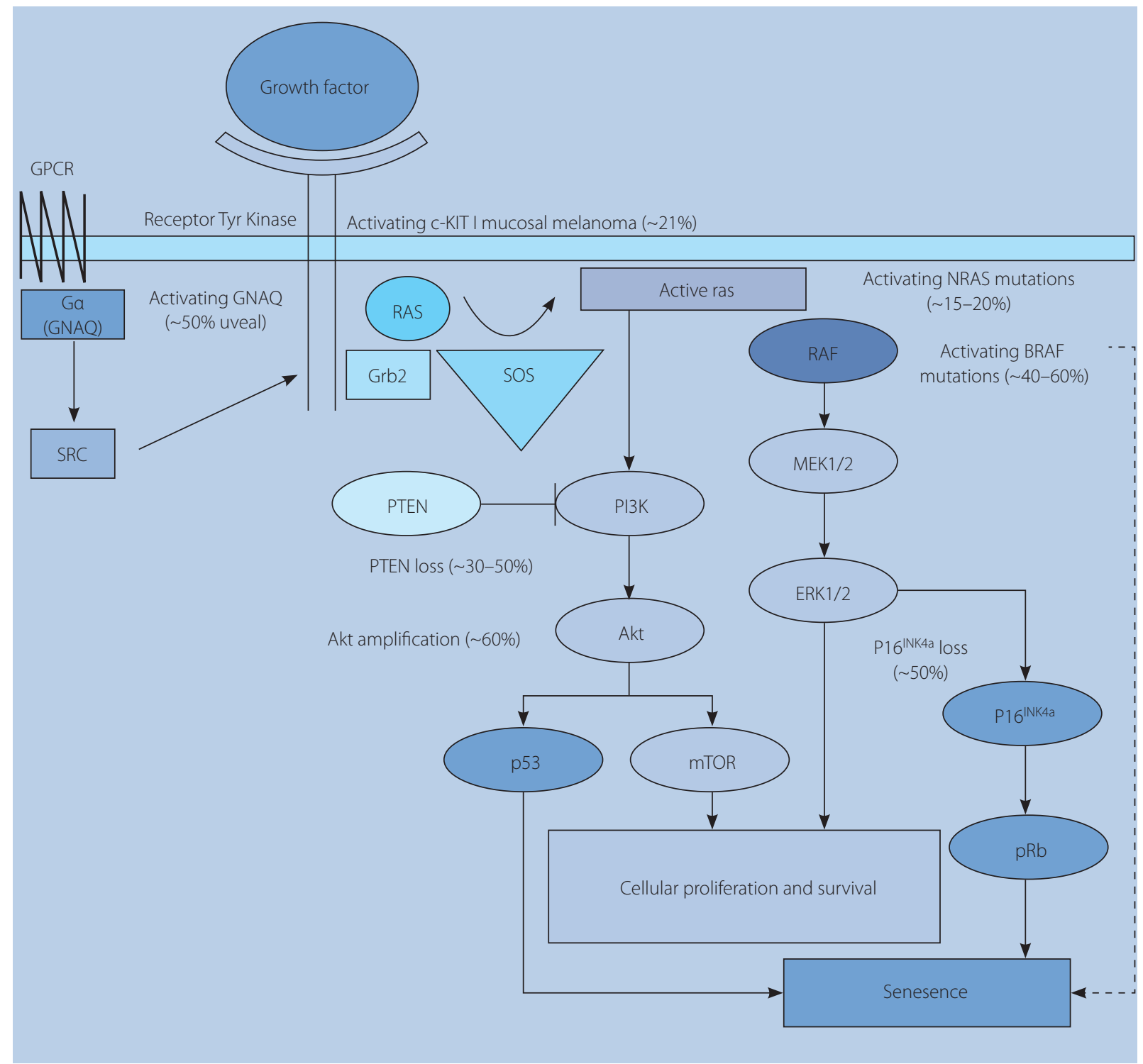

Figure 1. RAS pathway in melanoma cells 
conformation of BRAF protein, increasing its kinase activity, resulting in constitutive activation of the MAPK/ERK pathway. In the same place, but much less frequently, there are also other substitutions, e.g. V600K (7.8\%), V600R (1\%) (lysine and arginine substitution, respectively). In melanoma cells mutations also occur in other MAPK/ERK pathway genes, most often in the NRAS gene (13-25\%) [6, 7].

It is worth mentioning that mutations in the BRAF gene do not depend on UV radiation and are not sufficient to cause melanoma. The oncogenesis process is the result of many mutations in different genes. There are also other mutations in melanoma, e.g. in tumor suppressor genes TP53, PTEN and CDKN2A, as well as in the telomerase gene promoter [5]. Also important for melanoma development are mutations induced by UV radiation, which are characterized by transitions of C-> T nucleotides at the 3 ' end of pyrimidine dimers [8], such mutations occur in the above mentioned cancer suppressor genes, the telomerase gene and in p16. However, the issue of mutations induced by UV is complicated, they are different in different melanomas and molecular classification is based more on mutations that are not caused by UV rays [5]. Moreover, it is not easy to determine whether a given mutation was caused by UV, and it is not always certain [9].

In molecular terms, melanomas can be classified into 4 groups based on the mutations that occur in them: in RAS, in $B R A F$, in $N F 1$, and the fourth group consists of so-called triple-negative melanomas $[5,10,11]$. It is interesting that in primary melanomas mutations in RAS and BRAF genes never occur simultaneously. As for the MEK gene mutations (MAPK), there is no such good data as for mutations in many other genes, but since MEK inhibitors are also used in melanoma therapy together with BRAF inhibitors, MAP2K1 mutations occur in 5.38\% of patients with melanoma while MAPK1 mutations occur in $1.77 \%$ of patients with melanoma. Mutations in specific MAPK genes are often criteria for inclusion in clinical trials with MEK inhibitors (MEKi) [12].

BRAF protein normally acts as a dimer, but the V600E mutation causes it to act as a monomer. Inhibitors used in melanoma therapy, such as vemurafenib, have an effect on this monomer. Approximately $20 \%$ of patients with the BRAF V600E mutation do not respond to this drug [6]. The reasons for this are complex and may be due, among other things, to the heterogeneity of the cancer - e.g. not all of its cells have a target mutation, or to the loss of certain cancer suppressor genes, such as PTEN and NF1, which cause primary resistance to BRAFi/MEKi [6].

Similarly, after a few months of using BRAFi, melanoma cells become resistant to therapy, which may have different causes. In general, mutations that cause resistance act by increasing the frequency of RAF dimerization, although the most common cause of such resistance is reactivation of BRAF/ /MEK or another pro-proliferative signal transduction pathway. This reactivation may pass through ERK or other proteins. The reactivation can also be done by activating other RAFs, e.g. ARAF and CRAF. Another signal transduction pathway, such as PI3K-AKT-mTOR, can also be activated in the process of creating BRAFi/MEKi resistance. In inhibitor-resistant melanoma cells with BRAF V600E mutation there is a type- $\beta$ platelet-derived growth factor receptor (PDGFR $\beta$ ) over-expression, but other changes in these cells are also present. There is also an increased activation of the IGFR1 receptor (a receptor of insulin - like growth factor 1), which leads to activation of the PI3K-AKT-mTOR pathway. Finally, in some resistant cells, elevated levels of epidermal growth factor receptors are also found [2]. From the physiological point of view, in general, it is always about reactivation of the signalling pathway transmitting information about proliferation. It is not possible to determine the cause of the drug resistance in all cases.

Some causes of BRAFi/MEKi drug resistance are related to the BRAF gene itself. However, these are not typical mutations in the gene itself that change its structure, but those that cause changes in the splicing of the gene transcript. The result is a protein product again capable of forming dimers (which removes the activity of inhibitors that act on the monomer) or over-expression of this gene, resulting in more protein product that may result in the presence of dimers. Indirectly, RAS gene mutations can also influence dimerization [6]

\section{Mechanisms of primary resistance of melanoma to treatment with BRAF inhibitors}

Malignant melanomas are genetically very heterogenous. Moreover, they gain new mutations in the course of the metastasis process [13]. A significant proportion of melanoma tumor cells carry the BRAFV600E mutation and about $20 \%$ of patients with melanoma with this BRAFV600E mutation do not respond to treatment with BRAF inhibitors [14]. In addition, different cancer cells in the same patient may carry different mutations responsible for resistance to BRAF inhibitors [6]. The main proposed mechanisms of primary resistance to BRAF inhibitors, such as PTEN loss, RAC1P292S mutation, MAP3K8 over-expression, hepatocyte growth factor (HGF) secretion by stromal cells, NF1 suppressor gene loss and CCND1 amplification, are summarized in table I.

Future studies should provide a more detailed explanation of the mechanisms underlying the BRAF inhibitor resistance and the discovery of common mechanisms of resistance to different groups of chemotherapeutic agents. An important objective of the studies is to identify biomarkers of primary resistance to BRAF inhibitors so that the therapeutic response can be predicted before the onset of the therapy.

\section{Loss of the PTEN gene}

The PTEN (phosphatase and tensin homolog) gene is a suppressor gene - the protein encoded by this gene (phosphatidylinositol-3,4,5-trisphosphate 3-phosphatase - PTEN, MMAC1) is involved in cell cycle regulation. PTEN catalyzes PIP3 pho- 
Table I. The most important mechanisms of primary resistance to BRAF inhibitors

\begin{tabular}{|ll}
$\begin{array}{l}\text { Mutation/ other cause of } \\
\text { resistance }\end{array}$ & Proposed mechanism \\
\hline CCND1 amplification & $\begin{array}{l}\text { The CCND1 gene codes cyclin protein D1 - the key regulator of the cell cycle. Amplification } \\
\text { of the CCND1 gene and increase of cyclin D1 protein level maintain cell proliferation in the } \\
\text { presence of BRAF inhibitors. }\end{array}$ \\
\hline [22, 23] & $\begin{array}{l}\text { RAC1 } \\
\text { inh2925 mutation maintains MAPK signalling pathway transmission in the presence of BRAF }\end{array}$ \\
\hline [16, 51]
\end{tabular}

sphorylation in the 3'position of the inositol ring, which results in inhibition of the PI3K/AKT signalling pathway and finally inhibition of cell proliferation. The loss of the functional PTEN gene occurs in over $10 \%$ of melanoma cases and is one of the most common mutations responsible for BRAF inhibitor resistance [15]. Loss of PTEN expression leads to constitutive activation of the PI3K/AKT signalling pathway, which results in cell proliferation, cell growth and inhibition of apoptosis. The mechanism of resistance to BRAF inhibitors is inhibition of apoptosis induced by BIM protein (BCL2L11) [15].

\section{RAC1P29S gene mutation}

RAC1 protein (rac family small GTPase 1 cell migration-inducing gene 5 protein) is a GTPase, a regulator of the cell cycle, cellular adhesion, cell mobility (by interacting with the cytoskeleton) and cell differentiation. The P29S mutation in the RAC1gene, according to Watson and Li [16] study results, occurs in 3.3\% of cases of melanoma, so not very often. The presence of this mutation positively correlates with the mitotic index, the size of the lesion, as well as the occurrence of metastases [16]. However, the P29S mutation occurs in up to $20 \%$ of patients who do not respond to treatment with BRAF inhibitors [17]. Moreover, the presence of this mutation in melanoma cell lines causes resistance to BRAF inhibitors [16].

\section{MAP3K8 gene over-expression}

The MAP3K8 gene codes for the MAP3K8 protein (mitogen-activated protein kinase kinase kinase 8, otherwise COT, EST, ESTF, MEKK8, TPL2, Tpl-2, c-COT, AURA2). The MAP3K8 protein can activate the MAPK/ERK signalling pathway. Increased level of COT protein results in maintenance of proliferation despite BRAF inhibition. In the case of primary over-expression of MAP3K8, administration of BRAF inhibitors leads to even higher COT production and, as a result, to further intensification of proliferation $[18,19]$. MAP3K8 gene modifications occur in about 1.5\% of all patients with melanoma, often (in about 33\% of cases) they are present in Spitz nevi [20].

\section{Loss of NF1 protein function}

The NF1 gene encodes the neurofibromin protein (neurofibromin or neurofibromatosis-related protein - NF-1), which belongs to the group of proteins that activate GTPases. Neurofibromin is a negative regulator of RAS, the first protein of the MAPK signalling pathway. The loss of the functional NF1 gene product means an increase in RAS protein level and activation of the MAPK pathway, also in the presence of BRAF inhibitors. The loss of NF1 causes resistance to RAF, MAPK and BRAF inhibitors through constitutive activation of the MAPK kinase signalling pathway [21].

\section{CCND1 gene amplification}

The CCND1 gene (BCL1) codes for cyclin D1 (cyclin D1 or otherwise B-cell lymphoma 1 protein), the key protein responsible for cell cycle regulation (G1/S phase transition). BRAFi resistance due to CCND1 amplification is an example of BRAF inhibitor resistance associated with gene copy number change. In cells where the CCND1 gene was amplified, increased production of cyclin D1 occurs and as a result BRAF inhibition is not sufficient to inhibit proliferation [22]. Amplification of CCND1 is observed in more than $38 \%$ of melanoma samples, which indicates that a large group of patients is potentially resistant to BRAFi and could benefit from CDK4/6 inhibitor therapy $[23,24]$.

\section{Secretion of the hepatocyte growth factor by the stromal cells}

The hepatocyte growth factor (HGF) is a factor responsible for cell growth, mobility and morphogenesis. Pleiotropic activity of HGF occurs through its receptor, a transmembrane tyrosine kinase, encoded by the cMet proto-oncogene. An example of a mechanism of primary resistance to BRAF inhibitors, which 
is associated with the interaction of the tumor microenvironment, is HGF secretion by the stromal cells (including fibroblasts). HGF secretion leads to activation of the HGF receptor - MET protein - and activation of the MAPK/ERK and PI3K/ /AKT pathways (fig. 2). Their activation results in maintenance of proliferation in the presence of BRAF inhibitors $[25,26]$.

\section{Mechanisms of secondary resistance of melanoma to treatment with BRAF inhibitors}

During treatment, most patients develop secondary resistance to BRAFi/MEKi. The most frequent mechanism of secondary resistance to BRAFi/MEKi treatment is connected with reactivation of signal transduction through the MAPK/ERK pathway. Activation of this pathway may result from both the action of BRAF activating proteins activated by BRAF protein and the secondary activation of BRAF itself.

\section{Reactivation of signal transduction by MAPK/ /ERK}

Reactivation of signal transduction from the cell membrane to MAPK/ERK kinases (upstream reactivation) is a result of over-expression of receptors tyrosine kinases, which leads to cell division by activating ARAF and CRAF kinases in place of BRAF. Melanoma cells with the BRAF mutation V600E during treatment with BRAFi/MEKi can develop treatment resistance by switching the signal to different RAF isoforms (ARAF, BRAF or (RAF) and the resulting reactivation of transmission within the ERK pathway [14]. In melanoma cells there may be over-expression of ARAF or CRAF proteins while BRAF is blocked. ERK protein is a negative regulator of RAS protein. The BRAF inhibitor inhibits the growth of neoplastic cells by inhibiting the ERK pathway. The signal transduction blockage through the ERK pathway stops RAS regulation, inducing partial RAS activity. Activation of RAS leads to the creation of BRAFV600E dimers. BRAF inhibitors bind to one of the monomers, leading to the transactivation of the second monomer, not bound by the drug. Such activation of BRAF results in partial activation of signal transduction and contributes to the reduction of treatment efficacy $[27,28]$.

The MAPK/ERK signalling pathway may also be activated by the accumulation of activating mutations in the RAS gene as RAS promotes cell division by phosphorylating the ARAF and CRAF proteins to compensate for BRAF inhibition. After binding GTP, mutant RAS protein does not dissociate to an inactive form associated with GDP and is constantly activated. The PGD-related mutant RAS protein associated with PGD also promotes BRAF V600E dimerisation, reactivation of the ERK signalling pathway and ultimately influences the formation of resistance to BRAF inhibitors, as these drugs are exclusively associated with BRAFV600E monomers [29-31].

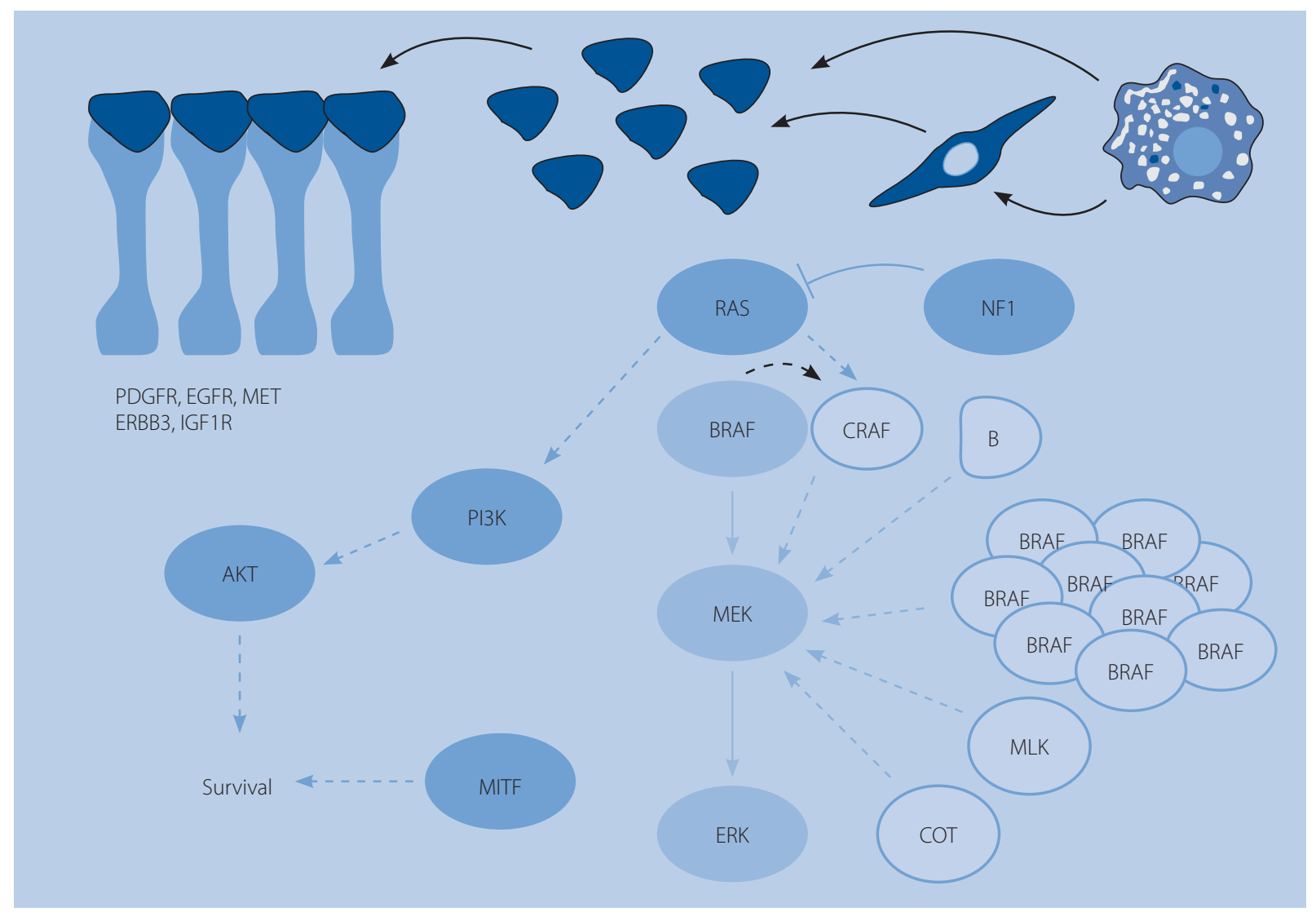

Figure 2. Activation of signalling transduction pathways in the development of drug resistance to BRAFi/MEKi 


\section{Activation of signal transduction below MAPK/ /ERK proteins}

The activation of signal transduction to target genes by the RAS-RAF-MEK-ERK signalling pathway can occur due to activating mutations in the genes for MEK1/MEK2 (mitogen-activated protein kinase 1/2) proteins. As a result of the activation of MEK proteins (downstream reactivation), the initiation of the signal transfer at the BRAF level is no longer necessary for the activation of target genes, thus eliminating the effect of BRAF inhibition [6].

\section{BRAF protein reactivation}

Reactivation of BRAF protein function may occur by many mechanisms, among which amplification of the mutant BRAF allele is frequent. The BRAF protein is over-expressed due to an increase in the number of gene copies. As a result of the presence of very many copies of BRAF protein in the cell, the inhibitor dose (the number of inhibitor molecules in the cell) is no longer sufficient (in proportion) to inhibit their activity. Increasing the amount of mutant BRAF V600E protein results from increasing the number of gene copies and may lead to spontaneous dimerization of this protein, reactivation of the ERK signalling pathway and become a cause of drug resistance. This type of resistance has been defined as dose-dependent. In in vitro studies, higher doses of vemurafenib lead to overcoming drug resistance [32].

In addition, the BRAF V600E variant, which is produced by alternative splicing of p61BRAF V600E, has been described in patients with secondary resistance to vemurafenib. This variant forms dimers independently of activation by RAS kinase, which makes BRAF inhibition ineffective, due to the above-mentioned BRAFi activity only towards BRAF V600E monomers. Alternative BRAF splicing isoforms appear to be formed as a result of mutations or epigenetic changes [33, 34].

\section{PI3K/AKT signalling pathway activation}

The PI3K/AKT signalling pathway communicates with the ERK pathway, and inhibiting one pathway can cause the other to become more active (figure 2). Blocking ERK signaling can lead to an adaptive PI3K/AKT overactivity that compensates for BRAF inhibition and drives resistance. Abnormal PI3K AKT signaling is a common feature of melanoma and causes resistance by stimulating alternative pathways that reduce dependence on ERK signaling. Mutations leading to increased activity of the PI3K/AKT pathway were identified in 22\% of melanomas with acquired BRAF inhibition resistance. It was shown that within a few days after the administration of BRAF inhibitors the expression of AKT protein was increased [35-37].

In preclinical studies it was initially hypothesized that during BRAFi/MEKi treatment there was a strong selection pressure on cells with gain of function mutations leading to increased PI3K AKT pathway activity in the presence of MAPK pathway inhibition. It was assumed that melanoma cells with such mutations will divide, because they have an advantage in terms of survival and proliferation when their metabolism is not affected by BRAF inhibition. In clinical observations, it is this intensive proliferation of cells with an activated AKT pathway that may explain the presence of high tumor mass and rapid progression in patients who responded to BRAF inhibition and then developed secondary resistance by this mechanism. Additionally, the PI3K AKT pathway is activated by growth factors that are associated with RTK, such as PDGFR- $\beta$ and IGF-1R. When BRAF is blocked by an inhibitor, tumor cells may increase the expression of PDGFR- $\beta$ and IGF-1R in a compensatory manner. This in turn leads to a persistent PI3K/AKT signaling that prevents apoptosis and promotes cell survival. Activated AKT phosphorylates as many as 9000 substrate proteins, thus regulating various processes such as cell survival, proliferation and migration, and affecting drug resistance.

The AKT substrate proteins include molecules such as: ASK1 (apoptotic signal kinase 1), Bim (B cell leukemia/lymphoma-2 interacting mediator of cell death), Bad (B cell leukemia/lymphoma-2 associated death agonist), MDM-2 (murine double minute-2), p21(p21 cyclin dependent kinase protein inhibitors, Cip1), XIAP (X-linked inhibitor of apoptosis), Foxo3a (forkhead box O3) and many others [38]. High expression of the above mentioned RTK receptors on the surface of melanoma cells is associated with acquired resistance to vemurafenib both in vitro and in vivo. In addition, PI3K and AKT activating mutations can increase the signal strength of the AKT signalling pathway. This in turn intensifies the anti-apoptotic signals and the regulation of expression of the most important proliferative genes described above. These changes allow melanoma cells to survive and replicate independently of BRAF inhibition, which clinically causes the acquired resistance $[6,37,39]$.

\section{Increased activation of the EGFR signalling pathway}

Increased expression and activation of the epidermal growth factor receptor (EGFR) may also be associated with BRAF or MEK inhibition resistance. Once activated, the EGRF complex formed by Grb2 and Sos proteins binds directly or by combining Shc adaptor protein with specific tyrosine residues on the receptor. This leads to conformational changes in the Sos protein, which can recruit and activate Ras-GDP.Then ERK-activated MAPK kinases eventually move to the nucleus to induce cell proliferation by phosphorylating specific transcription factors such as Elk1 and C-myc, [40]. Reduced SOX10 (sex determining region Y-box 10) activity described in some melanomas may lead to signaling via TGF- $\beta$ and consequently to increased expression of EGFR receptor gene and platelet-derived growth factor receptor (PDGFRB) [6, 41].

\section{Tumor microenvironmental activation}

The research on drug resistance focuses mainly on the mechanisms of drug resistance, which are a result of changes in the properties of cancer cells. It is now known, however, 
that disease progression and resistance to targeted BRAFi/ MEKi therapies are not exclusive derivatives of genomic and epigenetic cancer cell modifications.

The microenvironment of the tumor promoting MAPK inhibition resistance is important and this relationship is complex and includes interactions between the tumor and stromal cells, among others [42]. Recent studies have shown that macrophages and factors derived from fibroblasts clearly contribute to the development of resistance to MAPK pathway inhibitors. In the presence of fibroblasts, the adjacent melanoma cells acquire a differentiated, aggressive mesenchymal phenotype. After BRAFi treatment, these melanoma cells maintain a high level of phosphorylated ribosomal protein S6 (pS6), and thus active signaling through mTOR, which is suppressed in BRAFi-sensitive cells without contact with the stromal cells [43]. Activation of mTOR leads to phosphorylation and activation of the ribosomal kinase 56 p70 and eukaryotic protein binding factor $4 \mathrm{E} 1 \mathrm{E}$, thus promoting increased protein translation and cell growth. mTOR is a kinase that links cell stimulation with growth factors and nutrient availability with protein synthesis and cell growth [44].

In recent years it has been estimated that fibroblasts facilitate melanoma progression and two-way communication exists through direct contact between melanoma cells and fibroblasts. Additionally, melanoma cells respond to fibroblast' secretion of growth factors and cytokines promoting cell survival and growth, including TGF- $\beta$ and VEGF. Fibroblasts in contact with melanoma cells also release extracellular matrix components (e.g. laminin IV), which facilitate secondary migration (metastasis) of melanoma cells $[45,46]$. Within the tumor, fibroblasts show hyperactivation of the MAPK pathway, which results in a qualitative change in the extracellular matrix of the tumor by $\beta 1$ integrin and FAK kinase, which induce ERK, ensuring that melanoma cells can avoid effective treatment [47].

In melanoma, as a result of HGF secretion by stromal cells, resistance to BRAFi/MEKi may develop. HGF may bind RTK on the surface of melanoma cells, which will increase intracellular signaling promoting RAS expression, which ultimately leads to activation of the MAPK pathway. Moreover, it is known that HGF contributes to the development of resistance to BRAF inhibitor treatment by reducing the expression of genes encoding pro-apoptotic factors $[25,26]$.

The influence of fibroblasts on melanoma cells may differ significantly in elderly patients, because aging fibroblasts are more invasive. Recent studies have shown that aging fibroblasts increase the secretion of the sFRP2 factor - $\beta$-catenin inhibitor - which reduces the expression of MITF, leading to reduced expression of the APE1 redox regulator and makes melanoma cells more sensitive to oxidative stress, thus promoting secondary resistance to BRAF inhibition [48].

Macrophages of the tumor stroma secrete TNF-a factor, which promotes MITF expression depending on NF- $\beta$, which leads to BRAFi/MEKi resistance. Additionally, TNF-a has been proven to block apoptosis in cells in which BRAF is inhibited and to contribute to melanoma invasion and angiogenesis $[49,50]$.

\section{Summary}

The main mechanisms of resistance to BRAF inhibitors are:

1. Loss of inhibitory function of ERK kinase - BRAF inhibitor inhibits tumor growth by inhibiting the ERK pathway. This secondarily inhibits ERK negative feedback to RAS, which partially restores RAS activity. It leads to the formation of BRAFV600E dimers induced by RAS. BRAF inhibitors bind one and transactivate the other activate the other BRAF, reducing the effectiveness of BRAF inhibitor treatment.

2. Mutations activating the RAS gene - mutated RAS-GTP becomes constitutively active, increases BRAF V600E dimerization, reactivates the ERK pathway and secondarily promotes resistance to BRAF inhibitors that block only monomeric BRAFV600E.

3. Alternative splicing of BRAF V600E - the BRAF V600E splicing variant due to mutations or epigenetic changes can form dimers independently of RAS. This makes the BRAF inhibitor ineffective because it blocks only the monomeric BRAFV600E.

4. Over-expression of mutant protein BRAFV600E - an increased number of BRAFV600E copies in a cell (due to gene copy number increase) may also spontaneously favor BRAF V600E dimerization, reactivating the ERK pathway and causing treatment failure in some patients.

5. Activation of alternative RAF protein isoforms - BRAFV600E melanoma treated with BRAF inhibitors can acquire resistance by flexible switching between different RAF isoforms capable of reactivating the ERK pathway, increasing expression of ARAF or CRAF.

6. The over-expression of COT-COT protein, probably due to gene amplification or yet unidentified mechanisms, may reactivate MEK in the presence of BRAF inhibition, stimulating ERK signaling and drug resistance to BRAFi.

7. Mutations activating the MEK gene-mutations activating the MEK1/MEK2 gene make blocking the BRAF ineffective, because reactivation of MEK means that the MAPK/ERK pathway can still transmit a signal below BRAF, regardless of its inhibition.

8. Activation of the PI3K/AKT signalling pathway. Incorrect PI3K/AKT signaling is a frequent feature of melanoma. Blocking ERK signaling can lead to an adaptive PI3K/AKT overactivity that compensates for BRAF inhibition and promotes resistance.

9. Receptor tyrosine kinase (RTK) activation - the PI3K/AKT pathway is activated by growth factors that are associated with RTK, such as PDGFR- $\beta$ and IGF-1R. With the BRAF block, cancer cells can over-express RTK, leading to permanent PI3K/AKT signaling.

10. Activating mutations in PIBK/AKT genes - activating mutations in PI3KA AKT and AKT amplify AKT signaling, which in- 
creases anti-apoptotic signals and increases the expression of key proliferative genes, providing the cell with survival signals independent of BRAF.

11. EGFR signal path activation - EGFR activation induced by SOX10 suppression and increased TGF- $\beta$ pathway activity that causes cell ageing is reversed by BRAF/MEK inhibition.

\section{Conflicts of interest: none declared}

\section{Anna M. Czarnecka}

Maria Skłodowska-Curie Institute - Oncology Center Department of Soft Tissue/Bone Sarcoma and Melanoma ul. Roentgena 5

02-781 Warszawa, Poland

e-mail:am.czarnecka@coi.pl

Received and accepted: 3 Aug 2019

\section{References}

1. Davies $\mathrm{H}$, Bignell GR, Cox $\mathrm{C}$ et al. Mutations of the BRAF gene in human cancer. Nature. 2002; 417: 949-954.

2. Chan XY, Singh A, Osman N et al. Role played by signalling pathways in overcoming BRAF inhibitor resistance in melanoma. Int J Mol Sci. 2017; 18.

3. Bezniakow N, Gos M, Obersztyn E. The RASopathies as an example of RAS/MAPK pathway disturbances - clinical presentation and molecular pathogenesis of selected syndromes. Dev Period Med. 2014; 18: 285-296.

4. Molina JR, Adjei AA. The Ras/Raf/MAPK pathway. JThorac Oncol. 2006; 1:7-9.

5. Craig S, Earnshaw CH, Viros A. Ultraviolet light and melanoma. J Pathol. 2018; 244: 578-585.

6. Griffin $M$, Scotto $D$, Josephs $D H$ et al. BRAF inhibitors: resistance and the promise of combination treatments for melanoma. Oncotarget. 2017; 8: 78174-78192.

7. Mackiewicz J, Mackiewicz A. BRAF and MEK inhibitors in the era of immunotherapy in melanoma patients. Contemp Oncol (Pozn). 2018; 22: 68-72.

8. Winder $\mathrm{M}$, Viros A. Mechanisms of drug resistance in melanoma. Handbook of experimental pharmacology. 2018; 249: 91-108.

9. Brash DE. UV signature mutations. Photochem Photobiol. 2015; 91 : $15-26$.

10. Curtin JA, Fridlyand J, Kageshita T et al. Distinct sets of genetic alterations in melanoma. NEngl J Med. 2005; 353: 2135-2147.

11. Hayward NK, Wilmott JS, Waddell N et al. Whole-genome landscapes of major melanoma subtypes. Nature. 2017; 545: 175-180.

12. Consortium APG. AACR Project GENIE: Powering Precision Medicine through an International Consortium. Cancer Discov. 2017; 7: 818-831.

13. Swick JM, Maize JC, Sr. Molecular biology of melanoma. Journal of the American Academy of Dermatology. 2012; 67: 1049-1054.

14. Fedorenko IV, Paraiso KH, Smalley KS. Acquired and intrinsic BRAF inhibitor resistance in BRAF V600E mutant melanoma. Biochem Pharmacol. 2011; 82: 201-209.

15. Paraiso $\mathrm{KH}$, Xiang $\mathrm{Y}$, Rebecca VW et al. PTEN loss confers BRAF inhibitor resistance to melanoma cells through the suppression of BIM expression. Cancer research. 2011; 71: 2750-2760.

16. Watson IR, Li L, Cabeceiras PK et al. The RAC1 P29S hotspot mutation in melanoma confers resistance to pharmacological inhibition of RAF. Cancer research. 2014; 74: 4845-4852.

17. Van Allen EM, Wagle N, Sucker A et al. The genetic landscape of clinical resistance to RAF inhibition in metastatic melanoma. Cancer discovery. 2014; 4: 94-109.

18. Johannessen CM, Boehm JS, Kim SY et al. COT drives resistance to RAF inhibition through MAP kinase pathway reactivation. Nature. 2010; 468: 968-972.

19. Sharma V, Young L, Cavadas $M$ et al. Registered Report: COT drives resistance to RAF inhibition through MAP kinase pathway reactivation. eLife. 2016; 5.

20. Potentially Actionable MAP3K8 Alterations Are Common in Spitzoid Melanoma. Cancer discovery. 2019; 9: 574.
21. Whittaker SR, Theurillat JP, Van Allen E et al. A genome-scale RNA interference screen implicates NF1 loss in resistance to RAF inhibition. Cancer discovery. 2013; 3: 350-362.

22. Smalley KS, Lioni M, Dalla Palma M et al. Increased cyclin D1 expression can mediate BRAF inhibitor resistance in BRAFV600E-mutated melanomas. Molecular cancer therapeutics. 2008; 7: 2876-2883.

23. Wilson MA, Zhao F, Khare $S$ et al. Copy number changes are associated with response to treatment with carboplatin, paclitaxel, and sorafenib in melanoma. Clinical cancer research: an official journal of the American Association for Cancer Research. 2016; 22: 374-382.

24. Harris AL, Lee SE, Dawson LK et al. Targeting the cyclin dependent kinase and retinoblastoma axis overcomes standard of care resistance in BRAF (V600E) - mutant melanoma. Oncotarget. 2018; 9: 10905-10919.

25. Blum D, LaBarge S. Reproducibility project: Cancer B. Registered report: Tumour micro-environment elicits innate resistance to RAF inhibitors through HGF secretion. Elife. 2014; 3.

26. Straussman R, Morikawa $\mathrm{T}$, Shee $\mathrm{K}$ et al. Tumour micro-environment elicits innate resistance to RAF inhibitors through $\mathrm{HGF}$ secretion. Nature. 2012; 487: 500-504.

27. Villanueva J, Vultur A, Lee JT et al. Acquired resistance to BRAF inhibitors mediated by a RAF kinase switch in melanoma can be overcome by cotargeting MEK and IGF-1R/PI3K. Cancer cell. 2010; 18: 683-695.

28. Spagnolo F, Ghiorzo P, Orgiano L et al. BRAF-mutant melanoma: treatment approaches, resistance mechanisms, and diagnostic strategies. Onco Targets Ther. 2015; 8: 157-168.

29. Corcoran RB, Settleman J, Engelman JA. Potential therapeutic strategies to overcome acquired resistance to BRAF or MEK inhibitors in BRAF mutant cancers. Oncotarget. 2011; 2: 336-346.

30. Nazarian R, Shi H, Wang $\mathrm{Q}$ et al. Melanomas acquire resistance to BRAF(V600E) inhibition by RTK or N-RAS upregulation. Nature. 2010; 468: 973-977.

31. Romano E, Pradervand S, Paillusson A et al. Identification of multiple mechanisms of resistance to vemurafenib in a patient with BRAFV600Emutated cutaneous melanoma successfully rechallenged after progression. Clinical cancer research : an official journal of the American Association for Cancer Research. 2013; 19: 5749-5757.

32. Shi H, Moriceau G, Kong $X$ et al. Melanoma whole-exome sequencing identifies (V600E) B-RAF amplification-mediated acquired B-RAF inhibitor resistance. Nat Commun. 2012; 3: 724.

33. Poulikakos PI, Persaud Y, Janakiraman M et al. RAF inhibitor resistance is mediated by dimerization of aberrantly spliced BRAF(V600E). Nature. 2011; 480: 387-390.

34. Luco RF, Allo M, Schor IE et al. Epigenetics in alternative pre-mRNA splicing. Cell. 2011; 144: 16-26.

35. Shi $\mathrm{H}$, Hugo W, Kong $\mathrm{X}$ et al. Acquired resistance and clonal evolution in melanoma during BRAF inhibitor therapy. Cancer discovery. 2014; 4: 80-93.

36. Mendoza MC, Er EE, Blenis J. The Ras-ERK and PI3K-mTOR pathways: cross-talk and compensation. Trends Biochem Sci. 2011; 36: 320-328.

37. Shi $\mathrm{H}$, Hong $\mathrm{A}$, Kong $\mathrm{X}$ et al. A novel AKT1 mutant amplifies an adaptive melanoma response to BRAF inhibition. Cancer Discov. 2014; 4: 69-79.

38. Madhunapantula SV, Mosca PJ, Robertson GP. The Akt signaling pathway: an emerging therapeutic target in malignant melanoma. Cancer Biol Ther. 2011; 12: 1032-1049.

39. Das Thakur M, Stuart DD. Molecular pathways: response and resistance to BRAF and MEK inhibitors in BRAF(V600E) tumors. Clin Cancer Res. 2014; 20: 1074-1080.

40. Seshacharyulu P, Ponnusamy MP, Haridas D et al. Targeting the EGFR signaling pathway in cancer therapy. Expert Opin Ther Targets. 2012; 16: 15-31.

41. Sun $C$, Wang L, Huang $S$ et al. Reversible and adaptive resistance to BRAF(V600E) inhibition in melanoma. Nature. 2014; 508: 118-122.

42. Tape CJ, Ling S, Dimitriadi M et al. Oncogenic KRAS Regulates Tumor Cell Signaling via Stromal Reciprocation. Cell. 2016; 165: 910-920.

43. Seip K, Fleten KG, Barkovskaya A et al. Fibroblast-induced switching to the mesenchymal-like phenotype and PI3K/mTOR signaling protects melanoma cells from BRAF inhibitors. Oncotarget. 2016; 7: 19997-20015.

44. Karbowniczek M, Spittle CS, Morrison T et al. mTOR is activated in the majority of malignant melanomas. J Invest Dermatol. 2008; 128 : 980-987.

45. Li G, Satyamoorthy K, Meier F et al. Function and regulation of melanoma-stromal fibroblast interactions: when seeds meet soil. Oncogene. 2003; 22: 3162-3171.

46. Flach EH, Rebecca VW, Herlyn $M$ et al. Fibroblasts contribute to melanoma tumor growth and drug resistance. Mol Pharm. 2011; 8: 2039-2049. 
47. Hirata E, Girotti MR, Viros A et al. Intravital imaging reveals how BRAF inhibition generates drug-tolerant microenvironments with high integrin beta1/FAK signaling. Cancer Cell. 2015; 27: 574-588.

48. Kaur A, Webster MR, Marchbank K et al. sFRP2 in the aged microenvironment drives melanoma metastasis and therapy resistance. Nature. 2016; 532: 250-254.

49. Ruffell B, Coussens LM. Macrophages and therapeutic resistance in cancer. Cancer Cell. 2015; 27: 462-472.

50. Gray-Schopfer VC, Karasarides M, Hayward R et al. Tumor necrosis factor-alpha blocks apoptosis in melanoma cells when BRAF signaling is inhibited. Cancer Res. 2007; 67: 122-129.
51. Mar VJ, Wong SQ, Logan A et al. Clinical and pathological associations of the activating RAC1 P29S mutation in primary cutaneous melanoma. Pigment cell \& melanoma research. 2014; 27: 1117-1125.

52. Nissan MH, Pratilas CA, Jones AM et al. Loss of NF1 in cutaneous melanoma is associated with RAS activation and MEK dependence. Cancer research. 2014; 7 4: 2340-2350. 